\title{
Dissociation related to subjective memory fragmentation and intrusions but not to objective memory disturbances
}

\author{
Merel Kindt ${ }^{\mathrm{a}, *}$, Marcel Van den Hout ${ }^{\mathrm{b}}$, Nicole Buck ${ }^{\mathrm{b}}$ \\ ${ }^{a}$ Department of Clinical Psychology, University of Amsterdam, Roetersstraat 15, 1018 WB Amsterdam, \\ The Netherlands \\ ${ }^{\mathrm{b}}$ Department of Medical, Clinical and Experimental Psychology, Maastricht University, The Netherlands
}

\begin{abstract}
The present study was a replication of Kindt and Van den Hout (Behaviour Research and Therapy 41 (2003) 167) with several methodological adaptations. Two experiments were designed to test whether state dissociation is related to objective memory disturbances, or whether dissociation is confined to the realm of subjective experience. High trait dissociative participants $\left(N_{\text {exp. } 1}=25 ; N_{\text {exp. } 2}=25\right)$ and low trait dissociative participants $\left(N_{\text {exp. } 1}=25\right.$; $\left.N_{\text {exp.2 }}=25\right)$ were selected from normal samples $\left(N_{\text {exp.1 }}=372 ; N_{\text {exp.2 }}=341\right)$ on basis of scores on the Dissociative Experience Scale (DES). Participants watched an extremely aversive film, after which state dissociation was measured by the Peri-traumatic Dissociative Experience Questionnaire (PDEQ). Memory disturbances were assessed $4 \mathrm{~h}$ later (Exp. 1) or 1 week later (Exp. 2). Objective memory disturbances were assessed by a sequential memory task, items tapping perceptual representations (Exp. 1), or an open question with respect to the remembrance of the film (Exp. 2). Subjective memory disturbances were measured by means of visual analogue scales assessing fragmentation and intrusions. The two experiments provided a fairly exact replication of an earlier experiment (Behaviour Research and Therapy 41 (2003)
\end{abstract}

\footnotetext{
*Corresponding author. Fax: + 31206391369

E-mail address: m.kindt@uva.nl (M. Kindt).
} 
167-178), indicating a relation between dissociation and memory disturbances that appeared to be confined to the subjective experience of memory.

(C) 2004 Elsevier Ltd. All rights reserved.

Keywords: Dissociation; Memory disturbances; Subjective memory fragmentation; Intrusions; Objective memory fragmentation; Perceptual memory

\section{Introduction}

Clinical claims about an association between dissociation and memory disturbances have been around since 1900. Recently, this supposed relation was brought up again in research on PTSD. Trauma victims who develop chronic PTSD are supposed to suffer from intrusive memories and fragmented memories of the traumatic event (Foa \& Riggs, 1993; Ehlers \& Clark, 2000; Van der Kolk \& Fisler, 1995). It is assumed that dissociation is responsible for these memory disturbances. Moreover, it is argued that the detrimental effects of dissociation are due to disturbances in information processing of the traumatic event, resulting in memory disturbances (e.g. Spiegel \& Cardeña, 1991; Van der Kolk \& Fisler, 1995). There is indeed convincing evidence that dissociation at the time of the trauma and during the first month after the trauma is one of the best predictors of chronic PTSD (e.g. Bremner \& Brett, 1997; Ehlers, Mayou, \& Bryant, 1998; Freedman, Brandes, Peri, \& Shalev, 1999; Marmar et al., 1999; Murray, Ehlers, \& Mayou, 2002; Shalev, Peri, Canetti, \& Schreiber, 1996; Shalev et al., 1998; Tichenor, Marmar, Weiss, Metzler, \& Ronfeldt, 1996; Ursano et al., 1999). However, the finding that peri-traumatic dissociation is predictive of PTSD does not imply that disturbances in information processing are involved. Moreover, it should be noted that observation of memory disturbances in PTSD patients are mainly based on subjective memory assessments, that is clinical reports without experimental control of the memorised material. Note, however, that the subjective experience of memory does not necessarily overlap with the memory performance itself (see also Shimamura \& Squire, 1986). If the supposed memory disturbances are indeed the result of disturbances in information processing, not only subjectively assessed memory disturbances but also objectively assessed memory disturbances should be observed.

Recently, a laboratory study showed that dissociation was indeed related to memory fragmentation (Kindt \& Van den Hout, 2003). Although this observation is in line with clinical reports, the effect was restricted to subjective evaluation of the memory performance and no fragmentation was observed when memory fragmentation was assessed objectively by a sequential memory task. Only recently, several studies showed indeed a relation between dissociation and objectively assessed memory fragmentation (Harvey \& Bryant, 1999; Halligan, Michael, Clark, \& Ehlers, 2003). Note, however, that expressions of uncertainty with regards to memory and confusions were among others indices of memory fragmentation (see also Foa, Molnar, \& Cashman, 1995). In fact, these indices are also partly subjective evaluations of memory as opposed to objective memory performance. The present 
study examines further as to whether the relation between dissociation and memory disturbances can be observed in objectively assessed memory performance, or whether it is restricted to the realm of subjective experience (meta-memory).

Cognitive theory on information processing (Roediger, 1990; Graf \& Mandler, 1984) may be helpful to explain the supposed effect of dissociation on memory disturbances. First, dissociation during and after a traumatic event ('Peri-traumatic Dissociation', Marmar et al., 1994) may be understood as a state in which the subject processes the information primarily on a perceptual level without relating the event to existing knowledge (see also Ehlers \& Clark, 2000). This reminds of the processing modes described by Roediger (1990), who distinguished between data-driven (or bottom-up) and conceptually driven (or top-down) processing as opposing processing modes. A central premise is that the processing style determines the quality of the memory representations (Roediger, 1990). That is, data-driven processing results in perceptual and loosely interrelated memory representations of the original stimuli, whereas conceptually driven processing results in more abstract but integrated memory representations. It is suggested that peri-traumatic dissociation is associated with a mainly data-driven (bottom-up) processing style, and a relative lack of conceptually driven (top-down) processing, resulting in enhanced perceptual memory and memory fragmentation of the trauma (see also Ehlers \& Clark, 2000). More specifically, we suggest that dissociation is not only related to subjectively assessed memory disturbances such as intrusions and memory fragmentation but also to objectively assessed memory disturbances. These objectively assessed memory disturbances are operationalized in terms of a lack of sequential memory performance and a predominance of perceptual memories.

A second way in which dissociation during and after the traumatic event may result in memory disturbances is by suppression of the re-experiences or intrusions of the traumatic event. Suppression of intrusions may be dysfunctional in that it hinders the cognitive process indicated as 'elaboration', which occurs when schemata are activated in the presence of other mental events, making further new relationships (Graf \& Mandler, 1984). Such elaboration is required for a subject to conceptualise new experiences. Therefore, it is suggested that repeatedly reliving the traumatic experience, as opposed to suppressing the re-experiences, is a prerequisite for reprocessing the traumatic intrusions such that memory fragmentation is minimized (see also Ehlers \& Steil, 1995; Foa, Steketee, \& Rothbaum, 1989; Horowitz, 1976). Note, however, that although the tendency to dissociate is related to a tendency to suppress unwanted thoughts (Van den Hout, Merckelbach, \& Pool, 1996), suppression of intrusions could not account for the observed relation between dissociation and subjectively assessed memory fragmentation in a previous experiment (Kindt \& Van den Hout, 2003).

In sum then, cognitive theories predict that dissociation during and immediately after the trauma is related to memory disturbances. However, no evidence was found for this relation in a recent laboratory study (Kindt \& Van den Hout, 2003). That is, dissociation during an extremely aversive film was not related to objectively assessed memory fragmentation, but it was related to the subjective experience of memory being fragmented. Of course, limitations of the operationalisation of memory 
fragmentation may have been responsible for the absence of an effect in objective memory performance. That is, fragmentation in objective memory performance was solely operationalized by a sequential memory task, developed by Wegner, Quillian, and Houston (1996). We adapted this method by presenting $4 \times 5$ film clips from Passolini's 'Salò o le 120 giornate di Sodoma' in a fixed random order. The task of the participants was to write out the order in which these five films clips were originally presented in the film. This sequential memory task may not be valid in order to assess memory fragmentation. Using a similar sequential memory task as Wegner et al., the effect of suppression on memory fragmentation could also not be replicated by Rassin, Merckelbach, and Muris (1997). Another limitation of the laboratory study by Kindt and Van den Hout (2003) was that only memory fragmentation was operationalized and not perceptual memory.

The aim of the first experiment was to replicate the Kindt and Van den Hout (2003) study with two additions. First, disturbances in objective memory performance were not only operationalized by the sequential memory task but also by perceptual memory. With respect to perceptual memory, subjects were asked to describe several scenes in as much perceptual details as possible and the answers were independently judged in terms of percentage of perceptual features.

\section{Experiment 1}

\subsection{Method}

\subsubsection{Participants}

A total of 372 students volunteered to complete the Dissociative Experience Scale (DES; Bernstein \& Putnam, 1986) in order to assess trait dissociation. Following the cut-off recommended by Carlson, Putnam, Ross, and Torum (1993), two subsamples were selected from the total sample, that is, 25 subjects scoring above 30 $(M=37.8, \mathrm{SD}=5.2)$ and 25 subjects scoring below $10(M=3.0, \mathrm{SD}=1.4)$. Individuals who had a history of sexual or physical abuse were excluded from the study. The other measures were only obtained from the selected samples. The high DES group dissociated more during the film $(M=14.9, \mathrm{SD}=4.3)$, as was assessed by the Peri-traumatic Dissociative Experience Scale (PDEQ; Marmar, Weiss, \& Metzler, 1997) than the low DES group $(M=11.4$, SD $=3.4), t(48)=-3.2, p=$ .001 . Since the crucial issue in the present study is the effect of state dissociation on memory, the independent variable is the PDEQ. Mean age of the selected participants (15 males, 35 females) was 20.7 years $(\mathrm{SD}=2.5)$. Before the experiment started, participants provided written informed consent. They received a financial compensation for participation.

\subsubsection{Apparatus and materials}

The procedure and film were identical to the ones from Kindt and Van den Hout (2003). Also, the assessments were taken from Kindt and Van den Hout (2003), except that assessments of objective memory disturbances now also included 
questions measuring perceptual memory representations. These questions referred to certain scenes of the film. Moreover, also implicit memory tests were administered to the participants. However, the results of these tests will not be presented since the tests turned out to be invalid. That is, no main effects in memory performance were obtained for the experimental stimuli (i.e. presented film) in comparison to control stimuli (i.e. not presented in film) and such a difference was a logical pre-requisite for the interpretations of the presence/absence of dissociation effects.

\subsubsection{Stimulus material}

Stimulus material consisted of a 29-min compilation of 'Salò o le 120 giornate di Sodoma' produced by Passolini. This film contains scenes showing various kinds of sexual and physical abuse. The film looks like a documentary and the essence of the story and the chronological order were preserved in the 29-min compilation. Subjects were not acquainted with the film and did not realise that they saw only parts from the original. In the first phase, the different characters are introduced. This is followed by sexual and physical violence, which eventually ends in tortures.

\subsubsection{Trait dissociation}

Trait dissociation was measured by the DES (Bernstein \& Putnam, 1986). The DES is a 28 -item self-report questionnaire, whose items are scored on 100-mm visual analogue scales (VASs) that measures the tendency to dissociate. The higher the DES score, the stronger the tendency to dissociate (range: 0-100). The cut-off score recommended by Carlson et al. (1993) is 30, scores over 30 indicating dissociation. The reliability of the DES is high (Ross, Norton, and Anderson, 1988).

\subsubsection{State dissociation}

State dissociation was measured with the PDEQ Marmar et al., 1997). The PDEQ inquires about experiences that the subject recalls having had during the traumatic event. These dissociative experiences include altered time perception, depersonalisation and derealisation. Subjects are requested to rate each of the experiences on a 5point severity scale $(1=$ not at all, $5=$ extreme). The total score of the 7-item PDEQ ranges from 7 to 35 (see also Kindt \& Van den Hout, 2003).

\subsubsection{State anxiety}

State Anxiety was assessed by the Spielberger state anxiety scale of the State/Trait Anxiety Inventory (STAI; Spielberger, Gorsuch, \& Lusthene, 1970), with total scores ranging from 20 to 80 .

\subsubsection{Neuroticism}

Neuroticism was assessed by the Eysenck Personality Questionnaire (EPQ; Eysenck \& Eysenck, 1975), with total scores ranging from 0 to 22.

\subsubsection{Suppression and frequency of intrusions}

Suppression and frequency of intrusions were measured by $100-\mathrm{mm}$ VASs. 


\subsubsection{Arousal}

Arousal was measured by skin conductance level (SCL) with a Picker Schwartzer Skin Conductance Coupler (type EDA-48) using the constant voltage method $(0.5 \mathrm{~V})$. The coupler was connected to two Beckman $\mathrm{Ag}-\mathrm{AgCl}$ electrodes $(8 \mathrm{~mm}$ diameter), which were filled with isotonic gel and attached to the medial phalanges of the index- and middle finger of the non-dominant hand. SCL was measured before ( $2 \mathrm{~min}$ baseline) and during the film $(29 \mathrm{~min})$, and was timed and recorded (every $30 \mathrm{~s}$ ) by a microcomputer. Mean values of the SCL were calculated for the baseline and for the film.

\subsubsection{Objectively assessed memory disturbances}

The perceptual memory test consisted of questions referring to different scenes in the film like, e.g., "The prisoners had to behave like dogs and are also 'fed' like dogs" or "Ezio was caught in the act with the maid in his room", than, participants were asked to describe these scenes as detailed as possible. They were instructed to focus on the perceptual features of the scenes. The perceptual memory test comprised six items $($ alpha $=.87)$. One independent judge $(\mathrm{N}$. B. $)$ rated the answers by counting the percentage of perceptual utterances. An utterance was considered as perceptual in case it described solely a perceptual feature of the film and no conceptual inference had to be made. The utterances were not judged in terms of correctness. However, only the veridical utterances were scored. A second rater (M.K.), who was also blind to the dissociation scores, rated a random sample of 10 of the participants. Scores for the two raters showed high agreement. That is, the inter-rater reliability (ICC) was .93 based on 10 participants $(p<.01)$.

In the sequential memory task (see also Kindt \& Van den Hout, 2003), participants were shown four scenes comprising five clips (and $1 \times 5$ clips as a practice trial) from the film presented $4 \mathrm{~h}$ earlier, and they were told to write out the order in which these five clips had appeared in the film. They viewed the clips, each of which was presented for $10 \mathrm{~s}$, and then had to indicate the original order by describing the images. The five clips were shown in the same random order for all subjects.

\subsubsection{Subjective memory fragmentation}

Subjective memory fragmentation (meta-memory) was assessed by a $100-\mathrm{mm}$ VAS. Subjects had to indicate the "snap-shot" character of their recollections of the film.

\subsubsection{Procedure}

The selected participants (see "Participants") were tested twice: during a morning session and a session $4 \mathrm{~h}$ later in the afternoon. Subjects were tested individually and were alone during the film and the assessment. They sat in a room next to the equipment room, with a one-way screen. In the morning session, participants provided informed consent. The experimental procedure was explained by a female experimenter. Firstly, the experimenter attached the skin conductance electrodes to obtain a stabilisation period. Then, participants completed the state version of the STAI. After this, a 2-min baseline of the SCL was measured, followed by the 
presentation of the aversive film (29 min) during which the SCL was also measured. Subjects were instructed to concentrate on the film and not to divert attention because they would be asked some questions about the film. Only if the subjects could not handle parts of the film, they were allowed to look away. When they looked away, they had to push a yellow button. Hence, distraction was recorded by the computer, such that the experimenters could check which scenes were missed by each participant. These scenes were coded as missing data for the analyses of the memory tasks. Immediately after the film, subjects completed again the state version of the STAI and the PDEQ. The first test session lasted $45 \mathrm{~min}$.

In the second test session, $4 \mathrm{~h}$ later, subjects completed the VASs with respect to perceived memory fragmentation, frequency of intrusions, and suppression of intrusions. This was followed by the implicit memory tests, which are not further presented due to invalidity of the tasks. Then the subjects were administered the perceptual memory test and the sequential memory task. The session ended with the EPQ and an extensive debriefing, in which the background of the film was explained. The second test session lasted $90 \mathrm{~min}$.

\subsubsection{Data reduction and data analysis}

A manipulation check was executed with respect to STAI scores and SCL. It was investigated whether the film induced an increase in STAI scores and SCL, and whether a possible increase in STAI scores or SCL interacted with PDEQ scores. In that case, controlling for an increase in STAI scores or SCL would be required to test the hypotheses. Therefore, a median split (12) of the PDEQ scores was calculated. Subjects who scored above the median are referred to as the PDEQ + group $(M=16.7, \mathrm{SD}=3.2, N=23)$ and subjects scoring below the median are referred to as the PDEQ-group $(M=10.1, \mathrm{SD}=1.8, N=27)$. The STAI scores and the SCL were analysed with MANOVA's for repeated measures with Group (PDEQ + versus PDEQ-) as between-subjects factor and film effect (before versus after the film) as within-subject factor.

For the analysis of the Perceptual Memory test, mean percentages of memorised perceptual utterances were counted. The distribution parameters of the scores on the perceptual memory test were respectively, $M=87.5$, $\mathrm{SD}=5.5$, Kurtosis $=.40$, and Skewness $=-.64$. A Pearson correlation was calculated to test whether PDEQ scores were related to the performances on the perceptual memory test.

For the analysis of the Sequential Memory test, overall seriation scores for the sequence of the five events in each of the four scenes were calculated for each participant. Spearman correlations were computed between ranks of the original and recalled orders. Hence, the scores could range between -1 and 1, with 1 indicating the appropriate order. This measure reflects the resemblance between the original and retrieved overall order (see also Pellegrino and Hubert, 1982; Wegner et al., 1996; Kindt \& Van den Hout, 2003). Mean rank correlations were calculated for the four scenes. The distribution parameters of this variables were respectively, $M=.76$, $\mathrm{SD}=.11$, Kurtosis $=-.18$, and Skewness $=-.22$. In order to test whether PDEQ scores were associated with performance on the sequential memory task, a Pearson correlation was calculated. 
Furthermore, correlations were calculated between PDEQ, Subjective Memory Fragmentation, Suppression of Intrusions, Frequency of Intrusions, Neuroticism, State Anxiety change and SCL change. The distribution parameters of these variables were, respectively, PDEQ $(M=13.1, \quad \mathrm{SD}=4.2, \quad$ Kurtosis $=.09$, Skewness $=.72)$, Subjective Memory Fragmentation $(M=66.1, \quad \mathrm{SD}=22.7$, Kurtosis $=.04$, Skewness $=-.95)$, Suppression of Intrusions $(M=26.8, \mathrm{SD}=$ 28.4 , Kurtosis $=.48$, Skewness $=1.2)$, Frequency of Intrusions $(M=28.3, \mathrm{SD}=$ 28.4, Kurtosis $=-.24$, and Skewness $=.96)$, Neuroticism $(M=7.7, \mathrm{SD}=4.5$, Kurtosis $=-.08$, Skewness $=.22)$, State anxiety change $(M=12.1, \mathrm{SD}=9.5$, Kurtosis $=.93$, Skewness $=-.07)$ and SCL change $(M=.62, \mathrm{SD}=1.4$, Kurtosis $=$ 1.9 , Skewness $=-.24)$. Only Suppression of Intrusions and SCL change were not normally distributed (Kurtosis and/or Skewness $<-1$ or $>1$ ), and were therefore ranked. Only the predicted correlations were tested one-tailed, that is, between PDEQ and, respectively, Perceptual Memory scores, Sequential Memory performance, Subjective Memory Fragmentation, Suppression of Intrusions, Frequency of Intrusions, and Neuroticism, and between Suppression of Intrusions and Frequency of Intrusions (see Kindt \& Van den Hout, 2003).

\section{Results}

\subsection{Manipulation check}

As described in the subject section, subjects who scored high on the DES dissociated indeed more (PDEQ) during the film than subjects who scored low on the DES, $t(48)=-3.2, p=001$. The PDEQ scores varied from 7 to 24 .

State anxiety, as was assessed by the STAI, increased significantly during the film, $F(1,48)=106.7, p<.001$ (one-tailed), with a mean score of $35.0(\mathrm{SD}=6.5)$ before the film and a mean score of $47.1(\mathrm{SD}=11.5)$ after the film. Both the main effect for Group was significant, $F(1,48)=9.6, p<.01$ (two-tailed) as well as the interaction between Group and Film effect, $F(1,48)=13.4, p=.001$, indicating that the state anxiety increased more in the PDEQ + group (from $M=36.1, \mathrm{SD}=7.0$ before the film to $M=53.0, \mathrm{SD}=10.0$ after the film) than in the PDEQ- group (from $M=$ $34.0, \mathrm{SD}=6.1$ before the film to $M=42.1, \mathrm{SD}=10.4$ after the film).

There was also a film effect on SCL that increased significantly during the film, $F(1,47)=9.9, p<.01$ (one-tailed), with a mean baseline level of $4.2 \mu \mathrm{S}(\mathrm{SD}=1.6)$ and a mean level of $4.8 \mu \mathrm{S}(\mathrm{SD}=2.2)$ during the film. Neither the main effect for Group, $F(1,47)=1.1$, nor the interaction between Group and SCL change, $F(1,47)=.7$, were significant.

\subsection{Objective memory disturbances}

With respect to Objective Memory Fragmentation, no significant correlations were revealed between PDEQ and any of the memory tests. That is, Pearson correlations were respectively for the Perceptual Memory test and the Sequential 
Memory test .04 and -.09 . These results indicate that Dissociation during and immediately after the film was not related to Perceptual Memory or Memory Fragmentation for the aversive film material.

\subsection{Subjective memory disturbances}

The Pearson correlation between PDEQ and Subjective Memory Fragmentation was significant $(r=.24, p<.05$, one-tailed), indicating that subjects who reported more state dissociation during the film evaluated their memory of the film as more fragmentary. The other predicted correlations were also significant. That is, between PDEQ and Frequency of Intrusions $r=.37,(p<.01)$, between PDEQ and Suppression of Intrusions $r=.34(p<.01)$, between PDEQ and Neuroticism $r=$ $.38(p<.01)$, and between Suppression of Intrusions and Frequency of Intrusions $r=.74(p<.001)$.

Partial correlations were calculated to test whether Suppression of Intrusions, Frequency of Intrusions, Neuroticism, SCL increase, and State anxiety increase (STAI) explained the relation between PDEQ and Subjective Memory Fragmentation. Partial correlations were respectively, $r_{\text {partial,suppression }}=.18(p=.11)$, $r_{\text {partial,intrusions }}=.19(p=.096), \quad r_{\text {partial,neuroticism }}=.23(p=.06), \quad r_{\text {partial }, \mathrm{SCL}}=.20(p=$ $.09), r_{\text {partial,STAI }}=.16(p=.13)$. These partial correlations indicate that the observed relation between PDEQ and Subjective Memory Fragmentation is partly reduced by Frequency of Intrusions, Suppression of Intrusions and State anxiety increase. However, the reduction of explained variance is small.

In line with our previous study (Kindt \& Van den Hout, 2003), Subjective Memory Fragmentation was only related to state dissociation (PDEQ) and not to Trait Dissociation (DES), $r=.095$.

\section{Discussion}

This experiment replicated earlier findings on dissociation and memory fragmentation. No evidence was obtained for a relation between dissociation and objective memory disturbances, on either of the memory tests. Again, a relation was observed between dissociation and the evaluation of memory fragmentation, though this correlation was rather small. But given that the present data are a replication of our earlier study, strongly suggests that the relation between dissociation and subjective memory fragmentation is more than a chance finding.

On the one hand, there appears to be an effect of dissociation on subjective memory fragmentation and this time also on the frequency of intrusions. On the other hand, for the second time, there was no effect of dissociation on objective memory disturbances. As to the latter, there are some restrictions in the design that may account for the absence of a relation between dissociation and objective memory disturbances. Firstly, the operationalisations of perceptual memory and memory fragmentation used in the present experiment and in our earlier study forced the participants to focus on either perceptual aspects of the film or on the sequence 
of certain scenes in the film. This may have overshadowed individual differences in retrieval style, which may have been revealed in more open questions with respect to the remembrance of the film material.

Another restriction of our two experiments is that the memory performance was assessed only $4 \mathrm{~h}$ later. Prospective studies on the quality of intrusions after traumatic events showed that most trauma victims report intrusions immediately after a trauma and the frequency of intrusions diminish only with the passage of time. Moreover, from the observation that these intrusive memories are characterized by its sensoric quality, it is asserted that they are the result of data-driven processing. It may therefore be suggested that most trauma victims are initially prone to process a traumatic event mainly data-driven, and that it is a lack of a shift from data-driven to conceptually driven processing that is crucial in predicting the maintenance of intrusions and thus the development of chronic PTSD. In order to observe this processing shift, more time may be required between the film presentation and memory assessment. The second experiment dealt with those two restrictions by adapting the memory test and the time lag between the film presentation and the memory assessment.

\section{Experiment 2}

\subsection{Introduction}

The aim of this experiment was to replicate our earlier findings with two methodological adaptations in order to test as to whether the relation between dissociation and memory disturbances is observed by an objective measurement, or just restricted to subjective experience of fragmentation and intrusions. Moreover, it was tested whether the earlier findings would resist the passage of time. First, memory performance was assessed by only one test without cues about the retrieval mode (see also Roediger \& Bergman, 1998). Second, this memory test and the other variables from experiment 1 -i.e. Subjective Memory Fragmentation, Frequency of Intrusions, Suppression of Intrusions and Neuroticism - were measured 1 week after the film presentation. The other variables, i.e. PDEQ and STAI were measured in the first test session, similar to experiment 1 .

\section{Method}

\subsection{Participants}

Again, from a new sample of 341 students who completed the DES, subjects were selected using the same inclusion and exclusion criteria, resulting in 25 subjects scoring above $30(M=38.1, \mathrm{SD}=7.4)$ and 25 subjects scoring below $10(M=2.8$, $\mathrm{SD}=1.2)$. The high DES group dissociated more during the film $(M=13.9$, $\mathrm{SD}=3.5)$, as was assessed by the PDEQ than the low DES group $(M=11.2$, 
$\mathrm{SD}=4.2), t(48)=2.4, p=.01$. Mean age of the selected participants (eight males, 42 females) was 19.5 years $(\mathrm{SD}=1.8)$. Before the experiment started, participants provided written informed consent. They received a financial compensation for participation.

\subsection{Materials}

\subsubsection{Objectively assessed memory disturbances}

Participants were asked to think back to the film presented in the same room 1 week before and to write down what they could remember from the film. The writing assignment took $8 \mathrm{~min}$. The experimenter used a stopwatch to time the 8-min period and signed the participant to start and to finish the test via the intercom. Participants were not aware of the time limit for this test. Moreover, the 8-min period was determined by conducting a pilot study in which all students appeared to be finished in less than $8 \mathrm{~min}$. The narratives were divided into sentences and rated by two independent judges (M.K. and N.B.) who were unaware of the subject status and who did not ran the experiment. Narratives were judged on a 10-point Likert scale (from $1=$ 'not at all disorganized/fragmented' to $10=$ 'extremely disorganized/ fragmented' (see also Murray et al., 2002). Fragmentation was reflected in rambling from one subject to another, unfinished sentences, single words instead of sentences, and an inaccurate order of events. The ratings of the two judges showed high agreement, as is indicated by a high ICC of .93 $(N=50)$. Mean disorganization scores of the two judges were calculated.

\subsubsection{Procedure}

The second test session took place 1 week after the first test session in which the subjects were presented the film. Again, the session started with the VASs with respect to Subjective Memory Fragmentation, Frequency of Intrusions, and Suppression of Intrusions. This was followed by the memory test. The subjects were asked to read the instructions carefully and to sign the experimenter when they were ready to start. After $8 \mathrm{~min}$, the experimenter instructed the subject to finish the assignment. At the end of the memory test the subjects filled out the STAI, followed by the EPQ and an extensive debriefing.

\subsubsection{Data reduction}

For the analysis of the memory test, mean scores of the two judges on the fragmentation scales were calculated. The distribution parameters of the fragmentation scores were $M=5.8, \mathrm{SD}=2.6$, Kurtosis $=-.98$, and Skewness $=-.28$. The distribution parameters of the other variables were respectively, PDEQ $(M=12.6$, $\mathrm{SD}=4.1$, Kurtosis $=-.31$, Skewness $=.59$ ), Subjective Memory Fragmentation $(M=63.3, \mathrm{SD}=24.8$, Kurtosis $=.42$, Skewness $=-.96)$, Suppression of Intrusions $(M=13.8, \mathrm{SD}=19.2$, Kurtosis $=2.0$, Skewness $=1.8)$, Frequency of Intrusions $(M=12.2, \mathrm{SD}=16.9$, Kurtosis $=4.0$, and Skewness $=2.0)$, Neuroticism $(M=8.0$, $\mathrm{SD}=5.5$, Kurtosis $=.34$, Skewness $=.53$ ), State anxiety change during the memory test $(M=3.9, \mathrm{SD}=5.3$, Kurtosis $=.25$, Skewness $=.31)$. Suppression of Intrusions 
and Frequency of Intrusions were not normally distributed (Kurtosis and/or Skewness $<-1$ or $>1$ ), and were therefore ranked.

\section{Results}

\subsection{Objective memory disturbance}

In line with previous data, the Pearson correlation between PDEQ scores and Memory Fragmentation scores was far from significant, $r=-.07$.

\subsection{Subjective memory disturbances}

Again, the Pearson correlation between PDEQ and Subjective Memory Fragmentation was significant $(r=.30, p<.05)$, indicating that the earlier observed relation between dissociation and memory fragmentation is still present after 1 week. Furthermore, roughly the same pattern of data was revealed as in the previous experiments. That is, all Pearson correlations were significant: between PDEQ and Frequency of Intrusions $r=.56(p<.001)$, between PDEQ and Suppression of Intrusions $r=.51(p<.001)$, and between PDEQ and Neuroticism $r=.40(p<.01)$, and between Suppression of Intrusions and Frequency of Intrusions $r=$ $.73(p<.001)$. Also, the correlation between PDEQ and State Anxiety Increase during the memory test was significant, $r=.51(p<.001)$.

Partial correlations were calculated to test whether Suppression of Intrusions, Frequency of Intrusions, Neuroticism, and State Anxiety Increase (STAI) explained the relation between PDEQ and Subjective Memory Fragmentation. Partial correlations were respectively, $r_{\text {partial,suppression }}=.17(p=.12), \quad r_{\text {partial,intrusions }}=$ $.15(p=.15), r_{\text {partial,neuroticism }}=.21(p=.08), r_{\text {partial,STAI }}=.27(p=.03)$. These partial correlations indicate that neither neuroticism nor the reported increase in state anxiety during the memory test accounted for the relation between dissociation and perceived memory fragmentation. This time, however, the observed relation between PDEQ and Subjective Memory Fragmentation was reduced by both Frequency of Intrusions and Suppression of Intrusions.

In contrast with our previous experiments, Subjective Memory Fragmentation was not only related to State Dissociation (PDEQ), but also to ranked scores of Trait Dissociation (DES), $\quad r=.34(p<.01)$. However, a partial correlation showed that trait dissociation (DES) only marginally accounted for the relation between state dissociation (PDEQ) and Subjective Memory Fragmentation, $r_{\text {partial,DES }}=.20(p=.08)$.

\section{Discussion}

Findings were again replicated with respect to dissociation and memory fragmentation. For the third time in a row, state dissociation was not related to 
objective memory fragmentation, but it was related to the subjective experience of memory fragmentation (meta-memory). Apparently, the methodological adaptations did not affect these findings. It should be noted that the memory assessment at 1 week after the film presentation was a rather arbitrary choice. It is still possible that more time is required to show individual differences in objective memory fragmentation for aversive stimulus material. On the other hand, emotional arousal affected long-term memory with a testing session one week after training (Quevedo et al., 2003), and long-term memory becomes fully consolidated only several hours after acquisition (Cahill \& McGaugh, 1996). In addition, state dissociation explained also differences in frequency of intrusions and suppression of intrusions within this relatively short period of time. There is no a priori reason that the effect of dissociation during this film on objective memory disturbances would only be revealed after a longer period of time.

Interestingly, not only were the previous findings with respect to dissociation and subjective memory fragmentation also observed 1 week after film presentation, but the relations between dissociation and frequency of intrusions and suppression of intrusions were markedly significant and even stronger at 1 week assessment as compared to assessment at the same day. This adds to the validity of the present experimental paradigm to study the effect of dissociation on analogue PTSD symptoms.

\section{General discussion}

Dissociation during the aversive film is reliably associated with the subjective memory disturbances, but not with objective memory disturbances. Several operationalisations were used to test as to whether dissociation is related to objective memory disturbances, but with none of them the hypothesised association materialised. Although the present findings are not inconsistent with clinical reports, the data suggest that the claims on the disturbing effects of dissociation on information processing resulting in memory disturbances (e.g. Van der Kolk \& Fisler, 1995) are in fact unwarranted. A common objection raised by clinicians is that analogue studies using aversive films are not informative to understand the psychopathological mechanisms of PTSD. However, the highly significant relationships between dissociation and suppression of intrusions and frequency of intrusions indicate the validity of this paradigm to study analogue PTSD symptoms.

The predication that dissociation makes it difficult to retrieve trauma's as coherent narratives rests mainly upon subjective reports, and may be confined to metamemory. What does this mean then for the understanding of PTSD and processing of traumatic events? We started our introduction with the robust finding that dissociation during a traumatic event predicts reliably PTSD symptoms several months later. A common explanation for this effect is that dissociation disturbs information processing during and after traumatic events resulting in memory disturbances (Spiegel \& Cardeña, 1991; Marmar \& Weiss, 1996; Foa \& HearstIkeda, 1996; Ehlers \& Clark, 2000). Moreover, it is suggested that a lack of 
coherence in the trauma narrative is crucial in the maintenance of PTSD symptomatology. It may also be that the coherence of trauma is just an epiphenomenon of PTSD symptomatology instead of a causing or maintaining factor. Foa et al. (1995) observed that coherence in trauma narratives was increased after successful treatment. That is, group effects with respect to PTSD symptomatology and coherence of trauma narratives changed in the predicted direction after treatment. However, no individual relation was reported between decrement in PTSD symptomatology and increment in coherence. Moreover, a recent study could not find evidence for a relation between treatment effect in PTSD patients and coherence of trauma narratives, although the trauma narratives were generally more coherent after treatment (Van Minnen, Wessel, Dijkstra, \& Roelofs, 2002). Therefore, it may be concluded that coherence of the trauma narratives per se is not so crucial in PTSD (see also Brewin \& Holmes, 2003). The subjective evaluation of incoherence may just be a reflection of PTSD symptomatology. The presence of intrusions is one of the main characteristics of PTSD, and intrusions are qualified by their snapshot or fragmented character. Hence, only the presence of intrusions may result in the experience of memory fragmentation. Note that in the current experiment frequency of intrusions explained half of the variance of the relation between dissociation and subjective memory fragmentation.

Alternatively, dissociation may exert its influence on PTSD by disturbing the embedding or contextualisation of the traumatic event in autobiographical memory (see also Ehlers \& Clark, 2000; Brewin, 2001). Mandler (1979) distinguishes two dimensions of organisation to interpret a variety of different, often disparate appearing phenomena. First, the integration dimension refers to the degree to which the to-be-remembered items form functional units of thought and/or action; a highly integrated item is one in which the constituent elements of the response form a highly coherent unit. Second, the elaborative dimension refers to the degree of interrelatedness of the to-be-remembered item with other units in memory. Thus, integration measures the within-unit organisation, whereas elaboration measures the between-unit organisation. In trauma research, memory fragmentation has been investigated by focusing on the integrative dimension as opposed to the elaborative dimension. However, the elaborative dimension seems to be the most relevant dimension in understanding dysfunctional trauma processing, since it involves the formation and strengthening of associations between the trauma representation and other associated representations in memory. Note also that the degree of integration is primarily a function of the frequency of repetition. Thus, the observations that trauma narratives are more coherent after prolonged exposure therapy (Foa et al., 1995; Van Minnen et al., 2002) may just be an effect of rehearsal. Future research on trauma memory should focus on the contextualisation of the trauma or the interrelatedness of the to-be-remembered traumatic stimuli as opposed to the coherence of the trauma narrative per se.

In sum then, for many methodological and ethical reasons it is hard to devise experimental models of PTSD. Although analogue studies are of course restrictive, the strong relations between dissociation and analogue PTSD symptoms show the validity of these kinds of paradigms to study fundamental issues of PTSD. Both in 
very early and in recent views on trauma and memory, an association is postulated between dissociation and memory disturbances. The present two experiments provided a fairly exact replication of an earlier experiment (Kindt \& Van den Hout, 2003) and they suggest that the idea of the detrimental effects of dissociation on information processing resulting in memory disturbances should be modified. Yes, there is an association between dissociation and memory disturbances. But no, this association is not between dissociation and objective memory disturbances, but it appears to be confined to subjective experience of memory. Data suggest that memory fragmentation or perceptual memory is meta-memory phenomena. Just the presence of intrusions may result in perceived memory fragmentation of the traumatic event. On the other hand, objective memory fragmentation has been studied by assessing the within-unit organization of trauma narratives, whereas the assessment of between-unit organization may be more appropriate. In future studies, methodological refinements are required to assess the contextualisation of trauma memories as opposed to assess the coherence of trauma narratives per se.

\section{Acknowledgement}

We gratefully acknowledge Ilse Janssen and Yvonne Megens assisted in data collection.

\section{References}

Bernstein, E., \& Putnam, F. W. (1986). Development, reliability and validity of a dissociation scale. Journal of Nervous and Mental Disease, 174, 727-735.

Bremner, J. D., \& Brett, E. (1997). Trauma-related dissociative states and long-term psychopathology in posttraumatic stress disorder. Journal of Traumatic Stress, 10, 37-49.

Brewin, C. (2001). A cognitive neuroscience account of posttraumatic stress disorder and its treatment. Behaviour Research and Therapy, 39, 373-393.

Brewin, C., \& Holmes, E. (2003). Psychological theories of posttraumatic stress disorder. Clinical Psychology Review, 23, 339-376.

Carlson, E. B., Putnam, F. W., Ross, C. A., \& Torum, M. (1993). Validity of the Dissociative Experience Scale in screening for multiple personality disorder: a multicenter study. American Journal of Psychiatry, 150, 1030-1036.

Cahill, L., \& McGaugh, J. L. (1996). Modulation of memory storage. Current Opinion in Neurobiology, 6, 237-242.

Ehlers, A., \& Clark, D. M. (2000). A cognitive model of posttraumatic stress disorder. Behaviour Research and Therapy, 38, 319-345.

Ehlers, A., Mayou, R. A., \& Bryant, B. (1998). Psychological predictors of chronic posttraumatic stress disorder after motor vehicle accidents. Journal of Abnormal Psychology, 107, 508-519.

Ehlers, A., \& Steil, R. (1995). Maintenance of intrusive memories in posttraumatic stress disorder: a cognitive approach. Behavioural and Cognitive Psychotherapy, 23, 217-249.

Eysenck, H. J., \& Eysenck, S. B. G. (1975). Manual of the Eysenck Personality Questionnaire. San Diego, CA: Educational and Industrial Testing service.

Foa, E. B., \& Hearst-Ikeda, D. (1996). Emotional dissociation in response to trauma: an information processing approach. In L. K. Michelson, \& W. J. Ray (Eds.), Handbook of dissociation: theoretical, empirical, and research perspectives (pp. 207-222). New York: Plenum Press. 
Foa, E. B., Molnar, C., \& Cashman, L. (1995). Change in rape narratives during exposure therapy for posttraumatic stress disorder. Journal of Traumatic Stress, 8, 675-690.

Foa, E. B., \& Riggs, D. S. (1993). Posttraumatic stress disorder and rape. In J. Oldman, M. B. Riba, \& A. Tasman (Eds.), Annual Review of Psychiatry, Vol. 12 (pp. 273-303). Washington, DC: American Psychiatric Association.

Foa, E. B., Steketee, G., \& Rothbaum, B. O. (1989). Behavioral/cognitive conceptualization of posttraumatic stress disorder. Behavior Therapy, 20, 155-176.

Freedman, S. A., Brandes, D., Peri, T., \& Shalev, A. (1999). Predictors of chronic post-traumatic stress disorder: a prospective study. British Journal of Psychiatry, 174, 353-359.

Graf, P., \& Mandler, G. (1984). Activation makes words more accessible, but not necessarily more retrievable. Journal of Verbal Learning and Verbal Behaviour, 23, 553-568.

Halligan, S. L., Michael, T., Clark, D., \& Ehlers, A. (2003). Posttraumatic stress disorder following assault: the role of cognitive processing, trauma memory and appraisals. Journal of Consulting and Clinical Psychology, 71, 419-431.

Harvey, A. G., \& Bryant, R. A. (1999). A qualitative investigation of the organization of traumatic memories. British Journal of Clinical Psychology, 38, 401-405.

Horowitz, M. J. (1976). Stress response syndromes. New York: Aronson.

Kindt, M., \& Van den Hout, M. (2003). Dissociation and memory fragmentation: experimental effects on meta-memory but not on actual memory performance. Behaviour Research and Therapy, 41, 167-178.

Mandler, G. (1979). Organization and repetition: organizational principles with special reference to rote learning. In L. G. Nilsson (Ed.), Perspectives on memory research (pp. 293-327). Hillsdale, NJ: Lawrence Erlbaum Associates.

Marmar, C. R., Weiss, D. S., \& Metzler, T. J. (1997). The Peritraumatic Dissociative Experience Questionnaire. In J. P. Wilson, \& T. M. Keane (Eds.), Assessing psychological trauma and PTSD: a practioner's handbook (pp. 412-428). New York: Guilford Press.

Marmar, C. R., Weiss, D. S., Metzler, T. J., \& Delucchi, K. (1996). Characteristics of emergency services personnel related to peri-traumatic dissociation during critical incident exposure. Americal Journal of Psychiatry, 153, 94-102.

Marmar, C. R., Weiss, D. S., Metzler, T. J., Delucchi, K. L., Best, S. R., \& Wentworth, K. A. (1999). Longitudinal course and predictors of continuing distress following critical incident exposure in emergency services personnel. Journal of Nervous and Mental Disease, 187, 15-22.

Marmar, C. R., Weiss, D. S., Schlenger, D. S., Fairbank, J. A., Jordan, B. K., Kulka, R. A., \& Hough, R. L. (1994). Peritraumatic dissociation and posttraumatic stress in male Vietnam theater veterans. American Journal of Psychiatry, 151, 902-907.

Murray, J., Ehlers, A., \& Mayou, R. A. (2002). Dissociation and post-traumatic stress disorder: two prospective studies of road traffic accidents survivors. British Journal of Psychiatry, 180, 363-368.

Pellegrino, J. W., \& Hubert, L. J. (1982). The analysis of organization and structure in free recall. In C. R. Puff (Ed.), Handbook of research methods in human memory and cognition (pp. 129-172). New York: Academic Press.

Quevedo, J., Sant' Anna, M. K., Madruga, M., Lovato, I., de-paris, F., Kapczinsky, F., Isquierdo, I., \& Cahill, L. (2003). Differential effects of emotional arousal in short- and long-term memory in healthy adults. Neurobiology of Learning and Memory, 79, 132-135.

Rassin, E., Merckelbach, H., \& Muris, P. (1997). Effects of thought suppression on episodic memory. Behaviour Research and Therapy, 35, 1035-1038.

Roediger, H. L. (1990). Implicit memory. American Psychologist, 45, 1043-1056.

Roediger, H. L., \& Bergman, E. T. (1998). The controversy over recovered memories. Psychology, Public Policy and Law, 4, 1091-1109.

Ross, C. A., Norton, G. R., \& Anderson, G. (1988). The Dissociative Experience Scale: a replication study. Dissociation: Progress in the Dissociative Disorders, 1, 21-23.

Shalev, A. Y., Freedman, S., Peri, T., Brandes, T., Sahar, T., Orr, S. P., \& Pitman, R. K. (1998). Prospective study of posttraumatic stress disorder and depression following trauma. American Journal of Psychiatry, 155, 630-637. 
Shalev, A. Y., Peri, T., Canetti, L., \& Schreiber, S. (1996). Predictors of PTSD in injured trauma survivors: a prospective study. American Journal of psychiatry, 153, 219-225.

Shimamura, A. P., \& Squire, L. R. (1986). Memory and metamemory: a study of the feeling-of-knowing phenomenon in amnesic patients. Journal of Experimental Psychology (Learn. Mem. Cognit.), 12, $452-460$.

Spiegel, D., \& Cardeña, E. (1991). Disintegrated experience: the dissociative disorders revisited. Abnormal Psychology, 100, 366-378.

Spielberger, C. D., Gorsuch, R. L., \& Lusthene, R. E. (1970). Manual for the State-Trait Anxiety Inventory. Palo Alto, CA: Consulting Psychologists Press.

Tichenor, V., Marmar, C. R., Weiss, D. S., Metzler, T. J., \& Ronfeldt, H. M. (1996). The relationship of peritraumatic dissociation and posttraumatic stress: findings in female Vietnam theater veterans. Journal of Consulting and Clinical Psychology, 64, 1054-1059.

Ursano, R. J., Fullerton, C. S., Epstein, R. S., Crowley, B., Vance, K., Kao, T. C., \& Baum, A. (1999). Peritraumatic dissociation and posttraumatic stress disorder following motor vehicle accidents. American Journal of Psychiatry, 156, 1808-1810.

Van den Hout, M., Merckelbach, H., \& Pool, K. (1996). Dissociation, reality monitoring, trauma, and thought suppression. Behavioural and Cognitive Psychotherapy, 24, 97-108.

Van der Kolk, B. A., \& Fisler, R. (1995). Dissociation and the fragmentary nature of traumatic memories: overview and exploratory study. Journal of Traumatic Stress, 8, 505-525.

Van Minnen, A., Wessel, I., Dijkstra, T., \& Roelofs, K. (2002). Change in PTSD patients'narratives during prolonged exposure therapy: a replication and extension. Journal of Traumatic Stress, 15, 255-258.

Wegner, D. M., Quillian, F., \& Houston, C. E. (1996). Memories out of order: thought suppression and the disturbance of sequence memory. Journal of Personality and Social Psychology, 71, 680-691. 